\title{
Comparative Study of Output Factors of Small Field Sizes Dosimetry Systems
}

\author{
SAMAH K. ELSHAMNDY, M.Sc. ${ }^{\mathbf{2}}$; PAOLO FRANCESCON, Ph.D. ${ }^{\mathbf{2}}$; NINFA SATARIANO, Ph.D. ${ }^{\mathbf{2}}$ \\ CHIARA ORLANDI, Ph.D. ${ }^{\mathbf{2}}$; HASSAN ABOUELENEIN, Ph.D. ${ }^{\mathbf{3}}$ and AHMED M. AHMED, Ph.D. ${ }^{4}$ \\ The Departments of Radiotherapy and Nuclear Medicine, Faculty of Medicine, Sohag University, Sohag, Egypt ${ }^{1}$, \\ Medical Physics, Vicenza General Hospital, Vicenza, Italy ${ }^{2}$, Radiotherapy, Children's Cancer Hospital, 57357, \\ Giza, Egypt ${ }^{3}$ and Physics, Faculty of Science, Sohag University, Sohag, Egypt ${ }^{4}$
}

\begin{abstract}
Background: The use of small fields in radiotherapy techniques has swiftly increased, in detail in stereotactic treatments and large uniform or nonuniform fields, which are composed of small fields such as for intensity-modulated radiation therapy (IMRT).

Aim of Study: Compare output factors (OFs) of small fields using various detectors and investigate its suitability for small field relative and absolute dosimetry through exploring the measured OFs with implementation to $6 \mathrm{MV}$ photon beams by a Cyber Knife ${ }^{\circledR}$. Normalization of different detector responses for the same field configuration is accomplished via applying a Monte Carlo derived correction factor.

Materials and Methods: Detectors used in the study were PTW60019 MicroDiaond, Exradin W1 Scintillator, and CCO1 IBA ion chamber, and field factors were calculated using Alfonso formula. OFs for a CyberKnife were measured in circular fields with the diameters range from $5 \mathrm{~mm}$ to $60 \mathrm{~mm}$ and using water tank at a $1.5 \mathrm{~cm}$ depth and at $80 \mathrm{~cm}$ sourceto-axis distance.

Results: The OFs measured by Micro Diamond and Exradin W1 (PSD) were within the uncertainties of the M.C. simulations for all the beam cones. While the CCO1 IBA ion chamber was unstable responding.

Conclusion: PTW 60019 microdiamond and Exradin W1 provide a useful and possibly superior alternative to existing dosimetry systems for small fields, as they are inherently less susceptible to volume-averaging and perturbation effects than larger, air-filled ionization chambers. Ultimately, they are favorable in small-field dosimetries.
\end{abstract}

Key Words: Synthetic diamond - CCO1 IBA ion chamberExradin W1 Scintillator (PSD) - Small field dosimetry - Relative dosimetry - Absolute dosimetry.

Correspondence to: Dr. Samah K. Elshamndy, E-Mail: d.shamndy@gmail.com

\section{Introduction}

THE CyberKnife system is based on 4 main subsystems: A 6 degrees of freedom robot, a compact linear accelerator at which 12 circular cones made up tungsten corresponding with diameter range start from $5 \mathrm{~mm}$ up to $60 \mathrm{~mm}$ (i.e., 5, 7.5, 10, 125 , $15,20,25,30,35,40,50$, and $60 \mathrm{~mm}$ ) can be delivered for small fields, an X-rays system and a dedicated treatment planning system. The linac source is at $80 \mathrm{~cm}$ from the virtual isocenter (which is better said the origin of the coordinate system, since there's no real isocenter). Additionally, 100 positions can be assumed by the source on a sphere centered on this point, and from each position 12 directions can be assumed, leading to 1200 different beams. It worth to be mentioned that not all the directions will really be used, but by the different weighting of these beams highly conformal shapes can be achieved. Compared to conventional stereotactic radiosurgery systems, the CyberKnife allows using of noncoplanar and non-isocentric geometries enhancing the ability to avoid critical structures. However, single isocenter and multi-center strategies can be used.

In small fields, detector readings are affected by both volume-averaging and by the densities of the detector sensitive volume and surrounding components. To a slighter extent, atomic number also affects detector readings, via differences between photon spectra in broad and narrow fields. When evaluating the accuracy of dosimetric measurements it should be established whether any part of the detector sensitive volume lies within a distance lower than the radius where the lateral electronic equilibrium breaks down; and if so, whether the electron fluence will be greatly per- 
turbed by a detector of the size, density and composition used, and whether accurate correction factors are available to account for the resulting perturbation and volume-averaging. An optimal detector would provide the dose at a point would be energy independent and would require only a single calibration valid for all possible energies and irradiation scenarios.

Air-filled ionization chambers possess lower limited in term of size by the signal to noise ratio, which $0.01 \mathrm{~cm}^{3}$ volume requires for therapeutic dose levels to achieve a signal noise ratio of around 1000 . For such small chambers, radiation-induced stem currents and cable currents become very large in term of comparison to the signal. The OF values associated with diodes are significantly greater than $\mathrm{O}$ (the field factor) for fields lower than $10 \mathrm{~mm}$ in diameter and correction factors must be applied.

A promising technology commercialized by PTW is the micro Diamond (MD) [1,2]. Although MD is inferior to a point scintillator in both aspects, it provides superior water equivalence to diode detectors and smaller sensitive volume than airfilled microchambers. The first evaluation of microdiamond measured OF values using CyberKnife VSI circular fields are compared with respect to the mean of corrected diode and microchamber measurements. Consequently, the maximum difference (microdiamond over-response) of $1.9 \%$ at the $7.5 \mathrm{~mm}$ field size is registered [3]. A subsequent measurement comparison using CyberKnife, in which a corrected diode measurement was used as a reference, inferred a maximum over-response of $0.6 \%$ at the $7.5 \mathrm{~mm}$ field size [4] and an expanded version of that study has reported this to be increased to $1.3 \%$ [5]

At the smallest field size $(5 \mathrm{~mm})$ these studies report an over-response of $1.0 \%$ [3], $0.2 \%$ [5] and under response of $0.2 \%$ [4]. This detector has been considered in several previous studies to use other treatment devices [6-12] and have recorded an inconsistent behavior at small field sizes, from over-response of $5.0 \%$ [9] to under-response of $2.7 \%$ [10]. The complexity of these results applicability to CyberKnife relies on the differences in collimator design, beam quality, measurement depth and distance, definition of machine specific reference field, and presence or absence of a flattening filter, and also by the variety of empirical and numerical methods employed.

In this paper we have tested the response in small fields of these new type of dosimeters (microdiamond and scintillating detector) and consolidated technologies CC01 IBA ion chamber, by measuring the output factor. The yielded results present an assessment of the MD dosimetric properties in view of its application in small field reference dosimetry. Also, due to most of the published article lack a proper estimation of the uncertainty in the various steps involved in the determination of output factor and the correction factors we will study the uncertainty of our detectors.

\section{Patients and Methods}

It worth to be mentioned that all detractors used in this study are at Medical Physics Department, ULSS, Vicenza, Italy as a part of STEP programme scholarship that funded by the ICTP/IAEA (20182019).

\section{Detectors:}

\section{PTW 60019 Micro Diamond:}

Conventionally, Micro Diamond (MD) detectors are considered as a solid state type associated with small size and high response. In addition, their response is almost independent upon energy, i.e. they are water equivalent. Additionally, they also characterized by a very good directional response. The outer dimension of the device cap as well as of the diamond plate position was marked by using a white dashed line. Technically, the $7 \mathrm{~mm}$ overall diameter of the MD is reported, together with the lateral size of the $3 \times 3 \times 0.3 \mathrm{~mm}$ diamond plate and the $2.2 \mathrm{~mm}$ diameter of the top contact [2]. The active volume implanted in the diamond crystal has a cylindrical shape of $1.1 \mathrm{~mm}$ radius and length of $1 \mathrm{~mm}$, the reference point is on the detector axis. According to literature reviews and manufacturers recommendation for all measurements, the Micro Diamond dosimeters should be oriented with their axis parallel to the beam direction with the detector facing up with the gantry at zero degrees $[\mathbf{1 , 1 0 , 1 1}$, $13,14]$

\section{Exradin W1 Scintillator (PSD):}

The plastic scintillator of Standard Imaging includes a light guide and an optical detector. The W 1 is nearly water equivalent and suppresses the Cerenkov light with the Supermax two channel electrometer. In fact, PSD was composed of a cylindrical scintillating fiber (multicladding SCSF$78 \mathrm{M}$, Kuraray Co., Ltd., Tokyo, Japan) with a diameter of $0.5 \mathrm{~mm}$ and a length of $1.0 \mathrm{~mm}$ coupled with a PMMAoptical fiber (Super Eska SH-2001, Mitsubishi, Rayon Co., Ltd., Tokyo, Japan) with a diameter of $0.5 \mathrm{~mm}$ and a length of $5 \mathrm{~m}$ to guide the scintillation produced to a polychromatic charge-coupled device (CCD) (U2000c, Apogee Imaging System, Roseville, CA, USA). A light col- 
lection system was developed to maximize the signal-to-noise ratio (SNR) using an optical lens (Minolta MC Rokkor-X PG, f/\#=1.4, focal length $=50 \mathrm{~mm}$ ). Pair the W1 Scintillator with the SuperMAX Electrometer to effectively eliminate Cherenkov Effect without the need for extraneous hand calculations. The dosimetric data was evaluated via Standard Imaging's two channel SuperMax electrometer.

\section{CCO 1 IBA ion chamber:}

Ionization chamber detecting relies on using a CC01 chamber from IBA Dosimetry America (Bartlett, TN), which is connected to a model 206 electrometer from CNMC Instruments Inc. (Nashville, TN). The ionization chamber is characterized by an active volume of $0.01 \mathrm{~cm}^{3}$, with a length of $3.6 \mathrm{~mm}$, an outer electrode that made up of airequivalent plastic (C-552) with a diameter of 2 $\mathrm{mm}$, and a steel inner electrode $2.8 \mathrm{~mm}$ long. The output ratios were yielded using both positive and negative polarities. Stabilization is being allowed by the chambers each time after the polarity was reversed. Technically, to achieve this a preirradiating is being applied for the chambers with 10Gy followed by a background measurements after the pre-irradiation. Actually, the polarity setting refers to the potential of the outer electrode relative to that of the inner collecting electrode, i.e. the positive polarity point out to positive charge collection at the collecting electrode and negative polarity indicate to negative charge collection at the collecting electrode. A bias voltage of $300 \mathrm{~V}$ was implemented for all chambers that correspond to the voltage recommended in the chamber's specifications for these chambers. According to the compact design of the chambers, Jaffe plots for all of these chambers were often used to evaluate the suitability of the chosen voltage. The values at $300 \mathrm{~V}$ were observed to be within a band of linear fit between 1/M and 1/U. Additionally, the measurements were repeated with the implementation of $100 \mathrm{~V}$ for this chamber and the yielded results were consistent with the results presented here for $300 \mathrm{~V}$.

\section{Experimental setups:} following:

Measurements were performed through the

(i) A CyberKnife® Robotic Radiosurgery System (Accuracy Incorporated, Sunnyvale, CA, USA), at Medical Physics Department, ULSS, Vicenza, Italy; (ii) The performance of OF measurements are occurred in a PTW MP3 water tank with a spatial position accuracy of $- \pm 0.1 \mathrm{~mm}$, which was used for scanning all detectors, by positioning the
MD, CC01 IBA and Plastic Scintillator Detector (PSD) detectors were used with their stems parallel to the beam axis (parallel orientation). It worth to be mentioned that no bias voltage was applied to MD and $300 \mathrm{~V}$ was applied to CC01 IBA, according to the manufacturer's manual. The PTW TRUFIXR detector positioning system was used for MD to improve the depth positioning accuracy in the water phantom.

CyberKnife measurements were accomplished in a beam type of 6MV flattening-filter-free (TPR20 $/ 10=0.640$ at a field size of $60 \mathrm{~mm}$ in diameter), delivered at $800 \mathrm{MU}$ min-1 and collimated via using circular fixed tungsten cones. SSD $80 \mathrm{~cm}$ has been implemented for the under study field sizes, which characterized with nominal diameters of $60 \mathrm{~mm}$, $50 \mathrm{~mm}, 40 \mathrm{~mm}, 35 \mathrm{~mm}, 30 \mathrm{~mm}, 25 \mathrm{~mm}, 20 \mathrm{~mm}$, $15 \mathrm{~mm}, 12.5 \mathrm{~mm}, 10 \mathrm{~mm}, 7.5 \mathrm{~mm}$ and $5 \mathrm{~mm}$. In fact, the definition of the machine-specific reference field fmsr is the $60 \mathrm{~mm}$ collimator. A complete characterization of this treatment system is given in Kilby et al., [15] . Additionally, SDD of $80 \mathrm{~cm}$ is being used for OF measurements performance, with the detectors positioned at a depth of $1.5 \mathrm{~cm}$ in the water phantom.

\section{Measuring protocols and data analysis}

Actually, OF measurements were performed for all the field sizes previously described. The measured OF values are defined as $[\mathbf{8 , 1 2}]$

$$
\begin{gathered}
\text { fclin, } f m s r \\
\text { Qclin, Qmse }
\end{gathered}=M_{\text {Qclin }}^{\text {fclin }}
$$

Where $M_{Q c l i n}^{f c l i n}$ and $M_{Q m s r}^{f m s r}$ are the readings of the investigated detector for both the $f_{\text {clin }}$ and the $f_{m s r}$ fields respectively. Besides, $f$ and $Q$ are respectively the collimator size in millimeters and the beam quality. The suffixes clin and msr show the field of interest (clinical field) and the machinespecific reference (60mm for a CyberKnife system), respectively [16]. The considered approach consisted of performing a measurement with the reference cone $(60 \mathrm{~mm})$ before and after the measurements with the cones of interest $(5-50 \mathrm{~mm})$.

Each detector reading represents the average of five successive measurements that yielded after $100 \mathrm{MU}$ irradiation steps. Thus, all the measured OF measurements have been corrected with a view to take into account the dose per pulse dependence of the device response, as recommended by the manufacturer (PTW 2017). Therefore, the observed applied correction factors for all OF values were below one percent. 
The field factors were yielded according to the following formula:

$$
\begin{gathered}
f_{\text {clin, }} f_{m s r} \quad f_{\text {clin }} f_{m s r} \quad f_{\text {clin, }} f_{m s r} \\
\perp \text { clin, Qmse }
\end{gathered}
$$

According to the formalism proposed by [16], by using the previously mentioned definition of OFs in equation (1) and the $K_{Q c l i n}^{f}$ cins $f_{m s}$ correction factors, which is evaluated by MC calculations.

\section{Uncertainty evaluation:}

In fact, the associated uncertainty of the $\mathrm{OF}$ results, which was evaluated according to the IAEA CoP-483 dosimetry protocol, relies on two main contributions: the first is the establishment of the measurement conditions $(0.4 \%, 1$ standard deviation, SD), while the second is the reading of the dosimeter relative to beam monitor $(0.6 \%, 1 \mathrm{SD})$. Consequently, these results are quoted in the same IAEA protocol as well. Thus, global uncertainty in the OF ratio of $1 \%(1 \mathrm{SD})$ is evaluated. Possible effects, which come from the unsuitable spatial resolution of the detector and from the difficulty of a correct detector positioning in narrow fields, were not taken into the account of the evaluation process.

\section{Cerenkov light effect:}

It worth to be mentioned that one of the unavoidable consequences of using optical fiber under irradiation at high energies is the production of Cerenkov light, which is a type of visible radiation that is emitted when a charged particle moves through a transparent medium (e.g., water or certain plastics) faster than the relativistic speed of light in that medium. The correction of Cerenkov background was performed for the W 1 using the twochannel scheme recommended by the vendor [17], whereby the correction of readings is derived from the charge measurements in color channels 1 (C1) and $2(\mathrm{C} 2)$ as Gain * (C1-C2 $*$ CLR). CLR is the Cerenkov light ratio and it is obtained using measurements rely upon two different irradiated optical fiber lengths. The geometry of water phantom with the detector oriented vertically as for OF measurement was used to determine CLR as described by Morin et al., [18] and recommended by the vendor (Standard Imaging 2014). On the other hand, W 1 results were accomplished using the vendor supplied dual-channel SuperMAX electrometer (Standard Imaging). CLR calibration and measuring the OFs were repeated over a period of 2.5 months to assess reproducibility.

\section{Results}

\section{Measurements with PTW 60019 MicroDiamond $(M D)$ :}

Fig. (1) Represents a comparison between OF measurements of PTW 60019 MicroDiamond de-

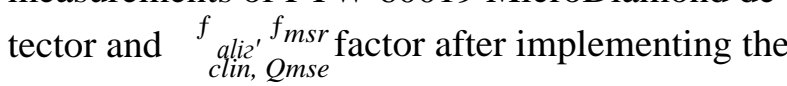
correction factor derived Monte Carlo (MC) simulation $K_{Q c l i n} \operatorname{lm}_{\text {Qmse }} f_{m s}$, which is reported in [19]. The figures demonstrate the slightly difference of the yielded OFs using MicroDiamond before and after applying the MC correction factor. For instance, the output factor with collimator diameter $5 \mathrm{~mm}$ is

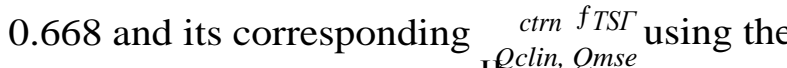
correction factor published by Francescon et al is 0.673 , while using (IAEA CoP-483) is 0.651 ; with collimator diameter $7.5 \mathrm{~mm}$ the output factor is

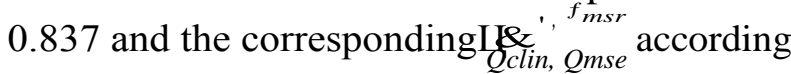
to the data of Francescon et al is 0.825 . The slightly difference is obvious by using the data of (IAEA TRS 483) in $\begin{gathered}\phi \text { i ciiri } f_{m s r} \text { by which it is } 0.822 . \text { The } \\ \text { Qclin, Qmse }\end{gathered}$ uncertainty have been calculated for correction factors of IAEA CoP-483 [19]. In general, there is a convention relies on this fact, which is there is no need to output correction factors to be applied to the PTW60019 MicroDiamond (MD) measurements for small field sizes. Consequently, the MicroDiamond is a good potential candidate for dosimeter of small field.

\section{Measurements with E xradin W1 Scintillator:}

Similarly, Fig. (2) demonstrates a Comparison between OF values and $\boldsymbol{O}_{\text {clin, }}^{\text {clin }} f_{m s r}$ for Exradin W 1 Scintillator detector with implantation to Francescon et al., correction factor [20]. The observed OF measurements using the Exradin W1 Scintillator possess very good agreement, with those calculated in two different Monte Carlo calculations mentioned before [21,20]. So, Exradin W1 Scintillator need the smallest correction factor for small field size that leads to consider the Exradin W 1 Scintillator one of our best choices to be implemented in small field dosimeters. Besides, the scintillation detector combines good water equivalence and a small sensitive volume.

\section{Measurements with IBA CCO1 ion chamber:}

In Fig. (3) Output factors were measured to all diameters using both polarities to evaluate the polarity effect at different diameters then we take the mean OF for each diameters. The output factor obtained using IBA CC01 ion chamber shows field 
size-dependent polarity effects at small field sizes in the axial orientation, which closely matched with Poppinga et al., $[\mathbf{2 2 , 2 3}]$. It worth to be considered, the effect of polarity for novel compact ionization chambers is suitably accounted in small field dosim-

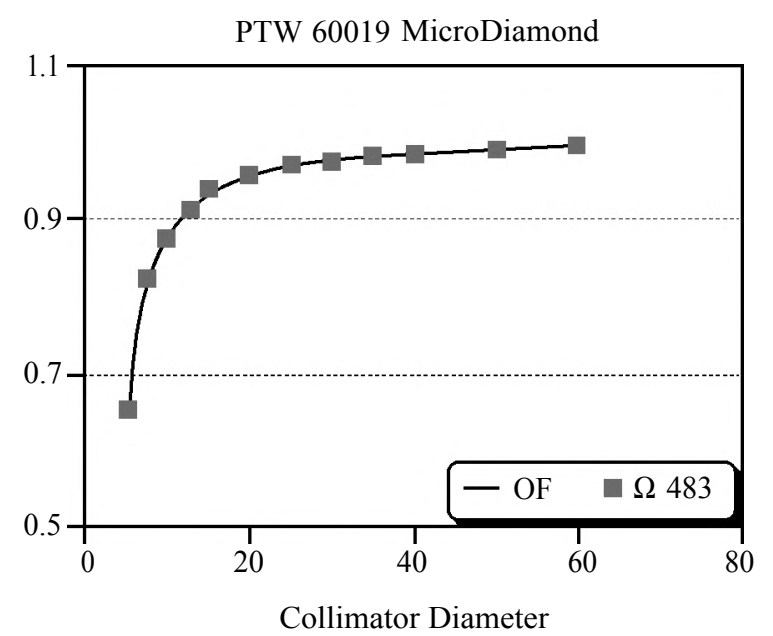

Fig. (1): Comparison between OF (Output Factor) and $\Omega 483$ in PTW 60019 MicroDiamond (MD) detector by using the correction by TRS483 [39] ( $\left.\begin{array}{c}f_{\text {clin, }} f_{m s r} \\ \text { Qclin, } \text { Omse }\end{array}\right)$ simplified $\Omega 483$ figure.

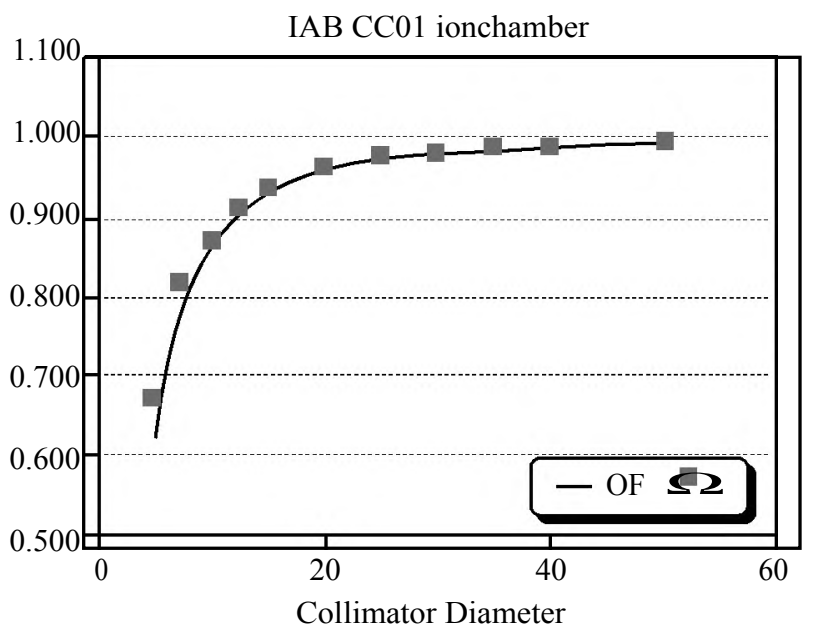

Fig. (3): Comparison between OF (Output Factor) and $\Omega$ in CC01 IBA ionchamber and using [19] correction factor $\left(0 \begin{array}{c}\text { clin, } f m s r \\ Q c l i n, Q m s e\end{array}\right)$ simplified $\Omega$ figure.

\section{Discussion}

The yielded results of several radiation detectors have been compared by measuring both output factors and field factors, i.e., $\begin{gathered}\text { ciin. } \mathbf{I} m s r \\ \text { cclin, } Q m s e\end{gathered}$ Actually, the field factor converts the absorbed dose in water for a machine-specific reference field fmsr to the absorbed dose in water for the clinical field $f_{\text {clin }}[\mathbf{1 6}]$. Recently used detectors have limitations to perform accurate small field dosimetry etry. Thus, it increases the possibility to study the chamber's design in order to minimize its polarity effect. The correction factors of small field outputs, which were computed according to TRS 483, have been reported for these investigated chambers.

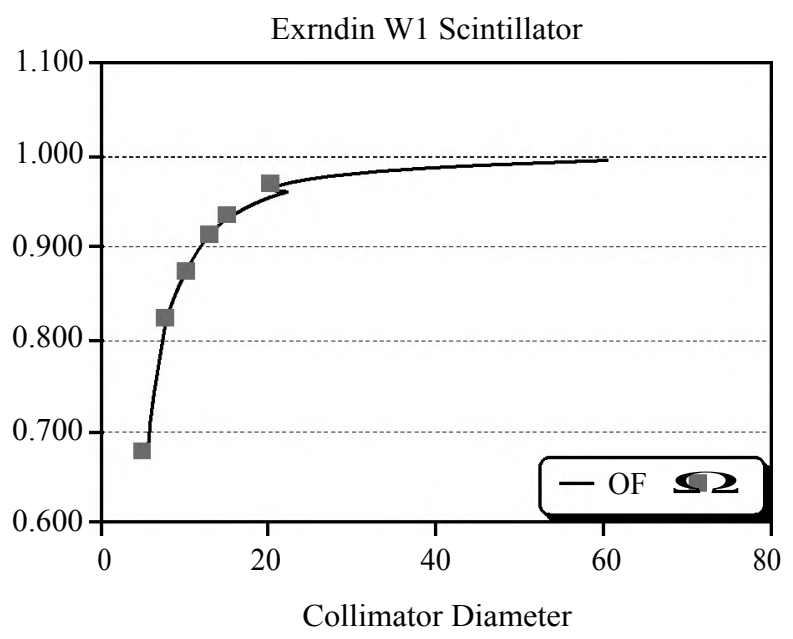

Fig. (2): Comparison between OF (Output Factor) and $\Omega$ in Exrndin W1 Scintillator detector using Francescon, et al., correction Facto [38] $f_{\text {clin, }} f_{m s r}$ Oclin, Omse simplified $\Omega$ figure.

measurements (fields $\leq 20 \mathrm{~mm}$ ) that was emphasized during the completion of this investigation. As broadly identified, the water nonequivalence of the silicon diodes is the responsible for the overresponse in the tails of the larger fields of CyberKnife systems as well as the small fields $<10 \mathrm{~mm}$.

From our result the CC01 IBA ion chamber unstable responded at the $5 \mathrm{~mm}$ cone because of a compensation for a volume averaging effect. Our data completely agree with published result of Francescon et al., [23]. Our findings show that small field factors, i.e., $\mathrm{q}_{\text {Qclin, }}^{\text {ciin. }}$ Qms $\mathrm{ms}$, can be accurately measured using water equivalent dosimeters such as PSDs, which provided results similar to those predicted by our independent Monte Carlo calculations. Exradin W1 Scintillator radiation detectors could be good candidates for reference radiosurgery dosimeters for water-based measurements such as total scatter factor, tissue phantom ratio, percent depth dose, and treatment delivery verification.

In the case of MicroDiamond detector, overall diameter sizes have observed to be identified as the most uniform response with corrections $\leq 1 \%$. Our results are associated with good agreement between MicroDiamond measurements and those made with other detectors. It worth to be mentioned that the corrections using simulations are based on the dimensions mentioned in [2]. The suitability 
of MicroDiamond for commissioning and routine use of CyberKnife is high. The associated measurements of this detector exhibit a good agreement of both DORs and profiles in the three directions, which confirm both the applicability and the suitability of Micro Diamond detector for clinical dosimetry.

The aim beyond this investigation is to find the optimal and applicable detectors to be used for measuring the output factors in small fields with CyberKnife ${ }^{\circledR}$, which is recently considered as a crucial question that needs to be answered. According to the output factor measurements, one suggested criterion should be taken into consideration is that the correction $\kappa_{Q \text { clin, Qmse }}^{\mathrm{f}^{\text {bll }},{ }_{1 \mathrm{~ms} r}}$ should remain $<5 \%$ for all field sizes [24]. From this stand point of view, our results emphasized that all used detectors meet this criterion for field size diameters $>20 \mathrm{~mm}$. In spite of that, for the smallest fixed collimators, only the PTW60019 MD and W 1 Exradin scintillation detector are suitable. Our yielded values are in high agreement with the published results by Francescon et al., [20]. Additionally, the Monte Carlo correction computed by Francescon et al., exhibit a good suitability to be implemented for all detectors under investigation according to the obtained standard deviation. Moreover, the mean values of the field output correction factors and the associated uncertainty have been estimated.

Actually, both side properties for each detector under study should be taken into consideration. Although both W 1 Exradin scintillator and PTW60019 microdiamond need significantly the smallest corrections for all measurement types, both of these detectors have disadvantages. Unfortunately, the major current practical limitation of the $\mathrm{W} 1$ detector does not interface with any commercially available plotting tank system. Thus, it can only be used for manual scanning that makes it impractical for anything except output factors measurement. Additionally, this detector exhibits a relatively large measurement for non-reproducibility after repeat set-ups, which it might be related to uncertainties in the Cerenkov correction obtained using the method of [18]. Similar CLR variability and output factor variations have been discovered in a larger measurement series using CyberKnife [5]. CLR inconsistencies have been also observed elsewhere and a dependency of CLR on the exact fiber orientation within the beam has been suggested [11].

According to our yielded results, the plastic scintillation dosimetry is suggested to be the favo- rable alternative to existing dosimetry systems for small fields, because the PSDs are less susceptible to volume-averaging and perturbation effects than larger, air-filled ionization chambers; therefore, PSDs may possess an inherent ability to determine output factors more accurately. Until the practical limitations of the W 1 Exradin scintillator are overcame, the synthetic microdiamond might be the closest candidate to be the optimal detector for small field dosimetry in a routine setting that is commercially favorable today.

\section{Conclusion:}

Concluding our discussion have been emphasized that the synthetic microdiamond detector (PTW60019 MicroDiamond) is considered as a promising technology for related investigations. From this perspective, it affords superior to smaller sensitive volume than air-filled microchambers, although this detector is inferior to an Exradin W 1 scintillator in both respects. By using it no essential corrections have to be implemented to the detector response for collimator diameter larger than about $10 \mathrm{~mm}$, since $\kappa_{-}^{\wedge l i n, Q m s e}{ }^{f_{\text {msr }}}$ is less than 0.1 in that range. Thus, the smallest fixed collimators only the synthetic microdiamond (PTW60019) and Exradin W 1 scintillation detector are suitable and applicable. The correction factors are much smaller for scintillation and synthetic microdiamond detectors, although both of these detectors exhibit other limitations, which make the microdiamond the more practical alternatively. Ultimately, the accurate relative dosimetry is viable by using the microDiamond and Exradin W 1 scintillation dosimeter for field sizes below $10 \mathrm{~mm}$.

\section{Acknowledgments:}

Kindly I want to acknowledge support from the ICTP/IAEA STEP programme for acceptance me and help me to take this chance to complete my Ph.D. work. Additionally, I cannot express enough thanks all Medical Physics group members in San Bortolo hospital.

\section{References}

1- I. CIANCAGLIONI, et al.: "Dosimetric characterization of a synthetic single crystal diamond detector in clinical radiation therapy small photon beams," Medical Physics, Vol. 39, No. 7, p. 4493, 2012.

2- M. MARINELLI, G. PRESTOPINO, C. VERONA, and G. VERONA-RINATI: "Experimental determination of the PTW 60019 microDiamond dosimeter active area and volume", Medical Physics, Vol., 43, No. 9, pp. 5205$5212,2016$.

3- A. CHALKLEY and G. HEYES: "Evaluation of a synthetic single-crystal diamond detector for relative dosimetry 
measurements on a CyberKnife???," British Journal of Radiology, Vol., 87, No. 1035, 2014.

4- S. RUSSO, et al.: "Multicenter evaluation of a synthetic single-crystal diamond detector for CyberKnife small field size output factors", Physica Medica, Vol., 32, No. 4, pp. 575-581, 2016.

5- L. MASI et al.: "CyberKnife beam output factor measurements: A multi-site and multi-detector study", Physica Medica, Vol., 32, No. 12, 2016.

6- G. AZANGWE, et al.: "Detector to detector corrections: A comprehensive experimental study of detector specific correction factors for beam output measurements for small radiotherapy beams", Medical Physics, Vol., 41, No. 7, 2014.

7- J.E. MORALES, S.B. CROWE, R. HILL, N. FREEMAN, J.V TRAPP and S.B. CROWE: "Dosimetry of conedefined stereotactic radiosurgery fields with a commercial synthetic diamond detector Dosimetry of cone-defined stereotactic radiosurgery fields with a commercial synthetic diamond detector", Vol., 111702, 2014.

8- P. PAPACONSTADOPOULOS, F. TESSIER and J. SEUNTJENS: "On the correction, perturbation and modification of small field detectors in relative dosimetry", Physics in Medicine and Biology, Vol., 59, No. 19, pp. 5937-5952, 2014.

9- A. RALSTON, M. TYLER, and P. LIU: “Over-response of synthetic microDiamond detectors in small radiation fields", Vol., 5873 .

10- J.M. LÁRRAGA-GUTIÉRREZ, P. BALLESTEROSZEBADÚA, M. RODRÍGUEZ-PONCE, O. A. GARCÍAGARDUÑO, and O.O.G. GALVÁN DE LA CRUZ: "Properties of a commercial PTW-60019 synthetic diamond detector for the dosimetry of small radiotherapy beams", Physics in Medicine and Biology, Vol., 60, No. 2, pp. 905-24, 2015.

11-T.S.A. UNDERWOOD, B.C. ROWLAND, R. FERRAND and L. VIEILLEVIGNE: "Application of the Exradin W1 scintillator to determine Ediode 60017 and microDiamond 60019 correction factors for relative dosimetry within small MV and FFF fields", Physics in Medicine and Biology, Vol., 60, No. 17, pp. 6669-6683, 2015.

12- D.J. O'BRIEN, L. LEÓN-VINTRÓ, and B. MCCLEAN: "Small field detector correction factors kQclin, Qmsrfclin, fmsr for silicon-diode and diamond detectors with circular $6 \mathrm{MV}$ fields derived using both empirical and numerical methods," Medical Physics, Vol., 43, No. 1, p. 411, 2016.

13- S. ALMAVIVA, et al.:"Synthetic single crystal diamond dosimeters for Intensity Modulated Radiation Therapy applications", Nuclear Instruments and Methods in Physics Research, Section A: Accelerators, Spectrometers, Detectors and Associated Equipment, Vol., 608, No. 1, pp. 191194, 2009.
14- P. MANCOSU, et al.: "Evaluation of a synthetic singlecrystal diamond detector for relative dosimetry on the Leksell Gamma Knife Perfexion radiosurgery system", Medical Physics, Vol., 42, No. 9, pp. 5035-5041, 2015.

15- W. KILBY, J.R. DOOLEY, G. KUDUVALLI, S. SAYEH and C.R. MAURER: "The CyberKnife ${ }^{\circledR}$ robotic radiosurgery system in 2010", Technology in Cancer Research and Treatment, Vol., 9, No. 5, pp. 433-452, 2010.

16- R. ALFONSO, et al.: "A new formalism for reference dosimetry of small and nonstandard fields", Medical Physics, Vol. 35, No. 11, pp. 5179-5186, 2008.

17- M. GUILLOT, L. BEAULIEU, L. ARCHAMBAULT, and S. BEDDAR: "A new water-equivalent 2D plastic scintillation detectors array for the dosimetry of megavoltage energy photon beams in radiation therapy", Vol., 38, No. Mlc, pp. 6763-6775, 2011.

18- J. MORIN, et al.: "A comparative study of small field total scatter factors and dose profiles using plastic scintillation detectors and other stereotactic dosimeters: The case of the CyberKnife," Medical Physics, Vol., 40, No. $1,2013$.

19- IAEA TRS 483, "Dosimetry of small static fields used in external beam radiotherapy: An IAEA-AAPM Internationa Code of Practice for reference and relative dose determination. Technical Report Series No. 483”, No. May, 2016.

20- P. FRANCESCON, W. KILBY, J. M. NOLL, L. MASI, N. SATARIANO and S. RUSSO: "Monte Carlo simulated corrections for beam commissioning measurements with circular and MLC shaped fields on the CyberKnife M6 system: A study including diode, microchamber, point scintillator, and synthetic microdiamond detectors", Physics in Medicine and Biology, Vol., 62, No. 3, pp. 10761095, 2017.

21- P. FRANCESCON, W. KILBY and N. SATARIANO: "Monte Carlo simulated correction factors for output factor measurement with the CyberKnife system-results for new detectors and correction factor dependence on measurement distance and detector orientation", Physics in medicine and biology, Vol. 59, No. 6, pp. N11-7, 2014.

22- D. POPPINGA: "The polarity effect of compact ionization chambers used for small fi eld dosimetry", Vol., 45, No. December, pp. 5608-5621, 2018.

23- P. FRANCESCON, W. KILBY, N. SATARIANO, C ORLANDI, and S. ELSHAMNDY: "The impact of interunit variations on small field dosimetry correction factors, with application to the CyberKnife system The impact of inter-unit variations on small field dosimetry correction factors, with application to the CyberKnife system", 2019.

24- H. PALMANS: "SP-0027: New IAEA-AAPM Code of Practice for dosimetry of small photon fields used in external beam radiotherapy", Radiotherapy and Oncology, Vol. 119, pp. S10-S11, 2016. 


\section{دراسة مقارنة لعوامل الخرج لأنظمة القياس الأشعاعى الهمى

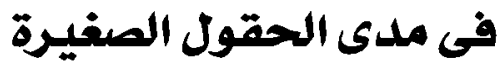

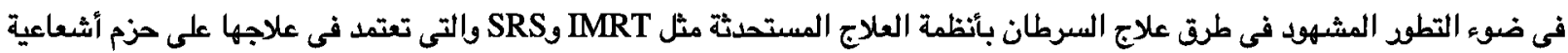

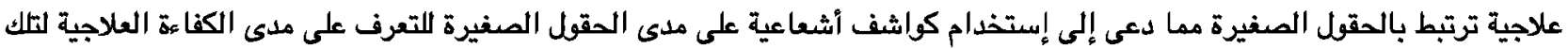

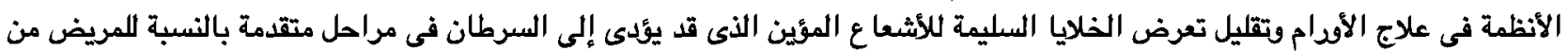

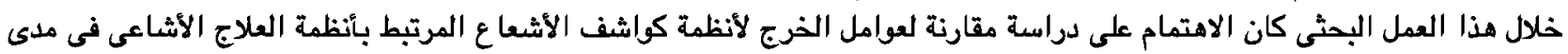

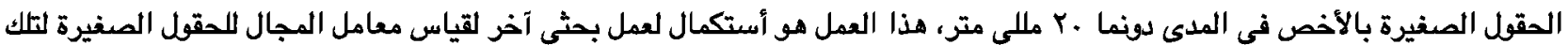
الأنظمة الحديثة العلاج للأشعاعى للأودام السرطانية وهو خاضع لتمويل وتوجيهات الوكالة الدولية للطاقة الذرية ومركز ICTP باليطاليا.

$$
\text { فى هذا البحث قد تم دراسة ومقارنة نتائج عوامل الخرج اللكواشف الأشعاعية الأتى أسمائها : }
$$

IBA CC01 ion chamer -1

Exradin W1 Scintillator $-Y$

PTW 60019 MicroDiamond (MD) $-r$

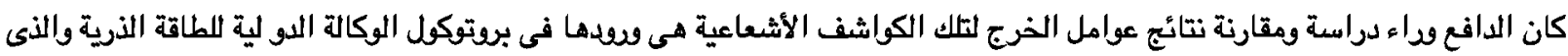

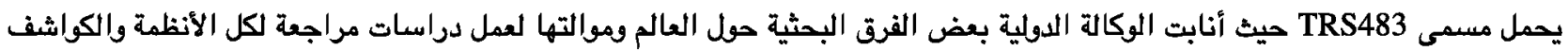

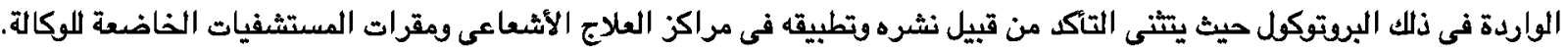

ومما وجدنا فى ما يخص نتائج الكاشف الأشعاعى المسمى IBA CC01 Ion chamer أن النتائج المتحصل عليها أختلفت بأختلاف القطبية

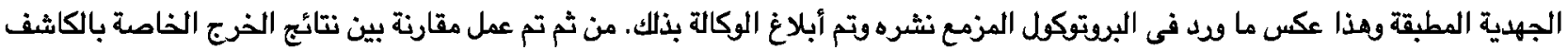

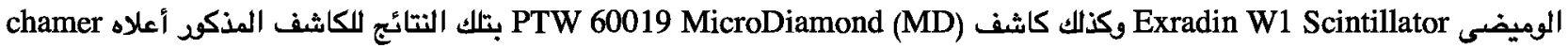

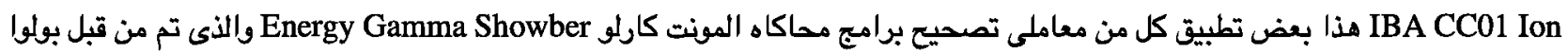

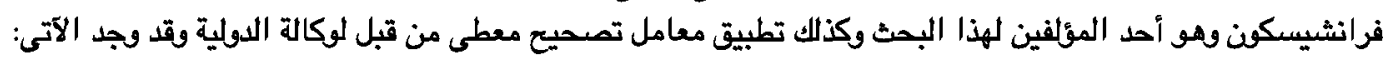

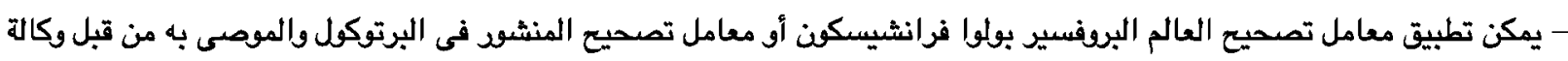

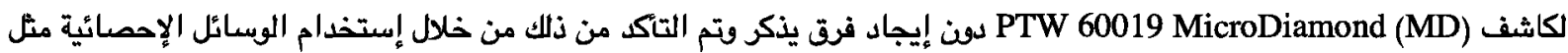
Confidence Interval, Uncertainty - تم التكد على التوصية بإستخدام كاشف PTW 60019 MicroDiamond (MD) فى أنظمة العلاج الأشعاعى فى مدى الصقل الصفيرة

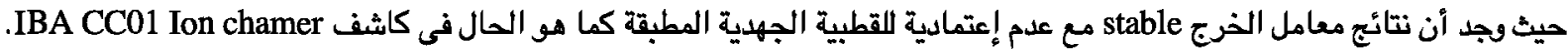

